\title{
Suppression of 2,4-dinitrochlorobenzene-induced atopic dermatitis by extract of Bacillus Calmette-Guerin
}

\author{
MINGLI SUN, SHUANG WANG, LIN ZHAO, HAISHAN ZHAO, \\ WEIFAN YAO, WANBAO JIN and MINJIE WEI \\ Department of Pharmacology, College of Pharmaceutical Science, China Medical University, \\ Shenyang, Liaoning 110001, P.R. China
}

Received June 6, 2013; Accepted November 28, 2013

DOI: $10.3892 / \mathrm{mmr} .2013 .1865$

\begin{abstract}
Bacillus Calmette-Guerin extract (BCGE) has been proven to be clinically effective for anaphylactic disease, infectious diseases and cancer. In this study, we investigated the effect of the intramuscular application of BCGE on 2,4-dinitrochlorobenzene (DNCB)-induced atopic dermatitis (AD). We established an AD model in BALB/c mice by repeated local exposure of DNCB to the ear and dorsal skin. Following intramuscular application of BCGE, the ear thickness, mast cell infiltration, serum total immunoglobulin $\mathrm{E}(\mathrm{IgE})$ and histamine level were measured. In addition, levels of interleukin (IL)-4, IL-13, interferon (IFN) $-\gamma$ and tumor necrosis factor (TNF) $\alpha$ in the ears were assayed. BCGE reduced AD symptoms based on ear thickness, dermatitis score, histopathological analysis and serum IgE levels. In addition, BCGE inhibited mast cell infiltration into the ear and elevation of serum histamine, increased IFN- $\gamma$ level and suppressed IL-4, IL-13 and TNF- $\alpha$ levels in the ears. Furthermore, BCGE attenuated the NF- $\kappa$ Bp65 expression in the nuclear extract of the ear tissue. Taken together, our results demonstrated that intramuscular application of BCGE exerts beneficial effects on the symptoms of AD suggesting that BCGE may be a candidate for the treatment of AD.
\end{abstract}

\section{Introduction}

Atopic dermatitis (AD) is a chronic, incurable, immune disease characterized by a relapsing course. Although the etiology of AD has not been fully described, genetic and environmental factors are believed to contribute to the underlying pathogenic mechanisms. AD is a biphasic inflammatory skin disease that

Correspondence to: Professor Minjie Wei, Department of Pharmacology, College of Pharmaceutical Science, China Medical University, 92 North 2nd Road, Heping, Shenyang, Liaoning 110001, P.R. China

E-mail: weiminjie1963@163.com

Key words: Bacillus Calmette-Guerin extract, atopic dermatitis, immunoglobulin E, interleukin-4 is provoked by an imbalance between T-helper (Th) 1 and Th2 immune responses. In particular, AD has been associated with the Th2 phenotype in which interleukin (IL)-4, IL-5, and IL-13 secretion and Th2-type cytokine-mediated IgE production are dominant (1). In addition, the accumulation of large numbers of eosinophils, mast cells and dendritic cells (DCs) has been observed in the epidermis and the dermis of patients with AD (2).

Numerous therapeutic trials have been performed in order to improve the symptoms of AD but with limited success. These include the prevention of Th2 responses, the enhancement of Th1 responses and the reduction of IgE concentration (3). Topical glucocorticoids are important and effective remedies for the treatment of AD. It is well established, however, that the prolonged use of a high dose of glucocorticoids causes a variety of side effects (4). Therefore, there is a great need for the development of new and effective therapies for AD.

Bacillus Calmette-Guerin extract (BCGE), including mainly lipopolysaccharide (77.8\%) and nucleic acid (16.67\%), is extracted from Bacillus Calmette-Guerin (BCG) by phenol. Lipopolysaccharide in BCGE is the main component of the cell wall in BCG, which is able to bind with toll-like receptors on the surface of dendritic cells, promoting the maturation of dendritic cells and secretion of IL-12, which causes the transformation from T0 to T1 and secretion of IFN- $\gamma$ (5). The toll ligand in cell walls of BCG is able to bind to toll-like receptors and then promote the maturation of DCs and the secretion of IL-12, which causes the transformation of $\mathrm{T} 0$ to $\mathrm{T} 1$ and the secretion of IFN- $\gamma$ (5). There is a rich $\mathrm{CpG}$ sequence in the genomic DNA of BCG, which is also named the immunostimulatory DNA sequence and is able to activate numerous immune effector cells. Yamamoto et al (6) demonstrated for the first time that DNA from Mycobacterium bovis (M.bovis) BCG activated natural killer (NK) cells and enhanced IFN- $\gamma$ production in mice. They also determined several sequences of 30-mer oligonucleotides that most potently augment the secretion of IFN- $\gamma$ and NK activity (7). Fujieda et al (8) demonstrated that the DNA fraction purified from $M$. bovis BCG inhibited IgE production and enhanced production of IFN- $\gamma$ and IL-12 in human peripheral blood mononuclear cells from normal and atopic donors. It may be assumed that the DNA fraction in 
the nucleic acid of BCGE was crucial for the relief of atopic symptoms by inhibiting IgE production. Recently, BCGE has been proven clinically effective for anaphylactic disease, infectious diseases and cancer in China (9). However, the effect of BCGE on AD was not elucidated. In the present study, we examined the effect of BCGE on AD lesions using the BALB/c model. Assessment was made by measuring ear thickness, histopathological changes including mast cell count, cytokine levels in the ear tissue, serum total $\operatorname{IgE}$ and histamine levels.

\section{Materials and methods}

Animals. Eight-week-old female BALB/c mice were purchased from the Center of Experimental Animals of the China Medical University (Shenyang, Liaoning, China) and all the procedures were performed in accordance with the NIH Guide for the Care and Use of Laboratory Animals (10). All animal experiments were performed in accordance with the Institutional Animal Ethics Committee and Animal Care guidelines for experimental animals at the China Medical University.

BCGE and reagents. BCGE was purchased from Wanma Pharmaceutical Co. Ltd. (Zhejiang, China), containing $14.663 \%$ nucleic acid, $10.005 \%$ water, $0.782 \%$ calcium, $0.270 \%$ inorganic phosphorus, $61.145 \%$ polysaccharides, $0.005 \%$ protein and $0.558 \%$ potassium. The extract was dissolved in saline for the experiments. DNCB was purchased from Sigma Chemical Co. Ltd. (St. Louis, MO, USA) and was dissolved in acetone/olive oil (1:3) solution.

Induction of dermatitis. Induction of $\mathrm{AD}$ was performed by using DNCB, as previously described (11), with minor modifications. The schematic experimental procedure was described in Fig. 1A. A total of $50 \mu 11 \%$ DNCB was applied to the outer and inner surfaces of mouse ears and $100 \mu \mathrm{l}$ of the same solution was applied to shaved dorsal skin on day 1 and 3. Then the same dose of $1 \%$ DNCB was applied once a week alternatively for 3 weeks.

Group and treatment. Mice were randomly divided into four groups: control group without DNCB application; AD group with DNCB application; $25 \mu \mathrm{g} / \mathrm{kg}$ BCGE-treated group with $25 \mu \mathrm{g} / \mathrm{kg}$ BCGE intramuscular administration and DNCB application; $75 \mu \mathrm{g} / \mathrm{kg}$ BCGE-treated group with $75 \mu \mathrm{g} / \mathrm{kg}$ BCGE intramuscular administration and DNCB application. The BCGE powder was dissolved in saline for intramuscular application. The same volume of saline was applied to the control and AD group instead of BCGE solution. On day 7, BCGE was intramuscularly applied every other day for 3 weeks. Following the last application of BCGE, the mice were sacrificed to investigate immunological and histological changes.

Ear thickness and clinical score of dermatitis severity. Ear thickness was measured twice a week using a micrometer (Mitutoyo Corporation, Kanagawa, Japan). The severity of dermatitis on the ear and back regions was evaluated twice a week. The development of i) erythema/hemorrhage, ii) scarring/dryness, iii) edema and iv) excoriation/erosion was scored as 0 (none), 1 (mild), 2 (moderate) or 3 (severe). The sum of the individual scores comprised the dermatitis score (12).

Histological observation. Ear samples were obtained $24 \mathrm{~h}$ following the final application of BCGE fixed in 10\% buffered formalin, embedded in paraffin and sectioned at $4 \mu \mathrm{m}$. For pathological observation, skin sections were stained with hematoxylin and eosin (H\&E). For the measurement of mast cell infiltration, skin sections were stained with toluidine blue and the number of mast cells in five sites was selected at random and counted.

Measurement of serum total IgE and histamine levels. Serum concentrations of $\operatorname{IgE}$ and histamine were determined by an IgE ELISA kit (Shibayagi Co., Ltd., Shibukawa, Japan) and histamine ELISA kit (Oxford Biomedical Research, Rochester, MI, USA), respectively, according to the manufacturer's instructions.

Measurement of cytokine levels in the ear. Tissue homogenate supernatant was acquired as previously described (13). Cytokine levels of IL-4, IL-13, IFN- $\gamma$ and TNF- $\alpha$ in the ear were determined by an ELISA kit (R\&D Systems, Wiesbaden, Germany) according to the manufacturer's instructions.

Measurement of $\mathrm{NF}-\kappa \mathrm{Bp} 65$ expression in the nuclear extract of the ear tissue. Nuclear proteins were extracted from the ear tissue using a nuclear protein extraction kit (Beyotime Biotechnology, Jiangsu, China). NF- $\kappa \mathrm{B}$ p65 expression in the nuclear extract was detected by western blotting.

Statistical analysis. Data are presented as the means \pm SD. Comparisons of more than two groups were made with a one-way analysis of variance ANOVA followed by Dunnett's test. $\mathrm{P}<0.05$ was considered to indicate a statistically significant difference.

\section{Results}

Effects of BCGE on DNCB-induced atopic dermatitis. As shown in Fig. 1B-D, repeated topical application of DNCB significantly increased ear thickness and dermatitis in $\mathrm{BALB} / \mathrm{c}$ mice compared with the control group. However, the application of BCGE reduced the DNCB-induced dermatitis (dermatitis index and ear thickness) since day 14 following DNCB application in a dose-dependent manner. Furthermore, histopathological data demonstrated that excessive inflammatory cells in the dermis were observed in the mice of the AD group. However, the application of BCGE reduced DNCBinduced inflammatory cell infiltration in a dose-dependent manner. These results indicated that BCGE suppressed the DNCB-induced dermatitis in a dose-dependent manner.

Effect of BCGE on mast cell infiltration, serum total IgE and histamine levels in the ear. To further investigate, we assessed the effects of BCGE on the infiltration of mast cells, an important effector cell and source of histamine involved in AD, into the ears. As shown in Fig. 2A and B, the infiltration of mast 
A

$$
\begin{aligned}
& 1: \text { BCGE } \\
& \square: \text { DNCB }
\end{aligned}
$$
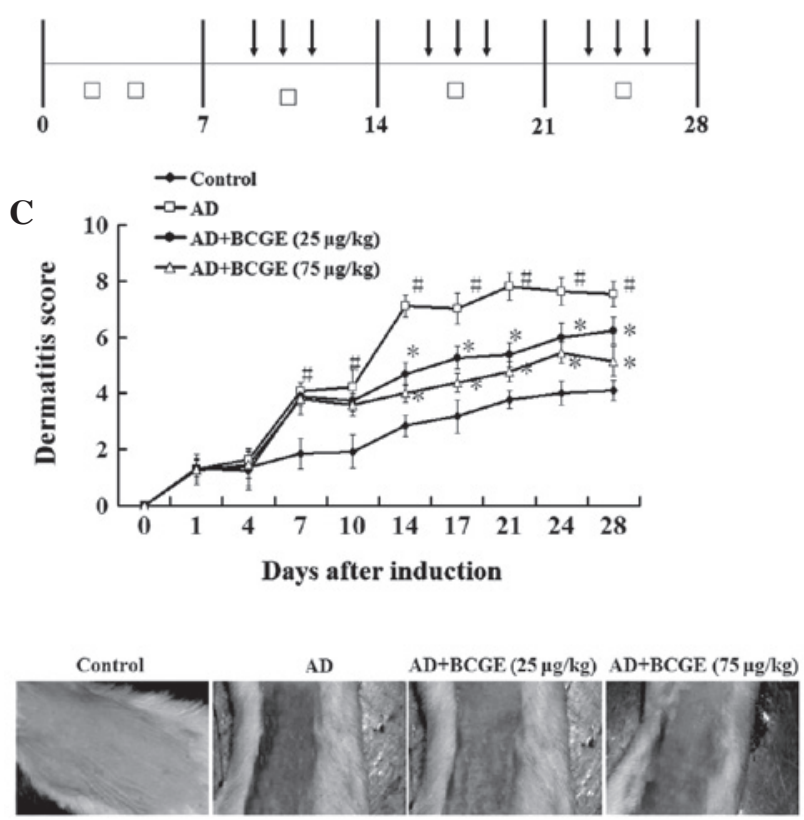

B

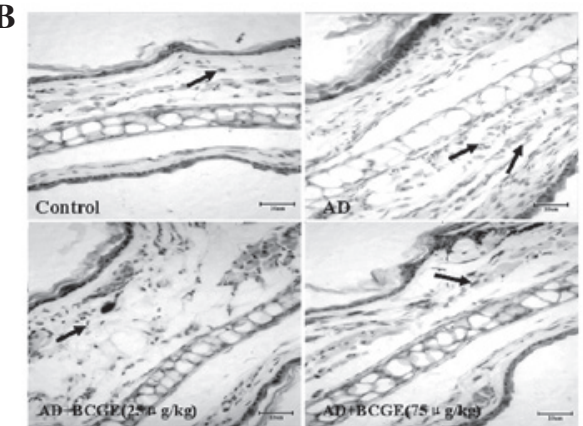

D

-Control

$\rightarrow-A D$

- AD+BCGE $(25 \mu \mathrm{g} / \mathrm{kg})$

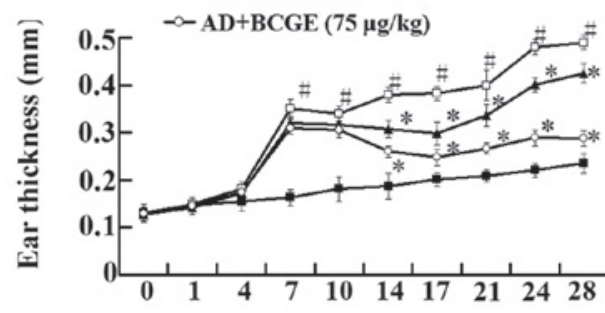

Days after induction

Figure 1. Effects of BCGE on the development of DNCB-induced AD in BALB/c mice. (A) Experimental schedule for the induction of dermatitis. (B) Histopathological features of skin lesions. (C) Dermatitis score was assessed using the criteria described in the materials and methods section. (D) Ear thickness was measured with a dial thickness gauge. Values are expressed as the means $\pm \mathrm{SD} .{ }^{~} \mathrm{P}<0.05$ vs. control group. ${ }^{*} \mathrm{P}<0.05$ vs. AD group. BCGE, Bacillus Calmette-Guerin extract; DNCB, 2,4-dinitrochlorobenzene; AD, atopic dermatitis.

A

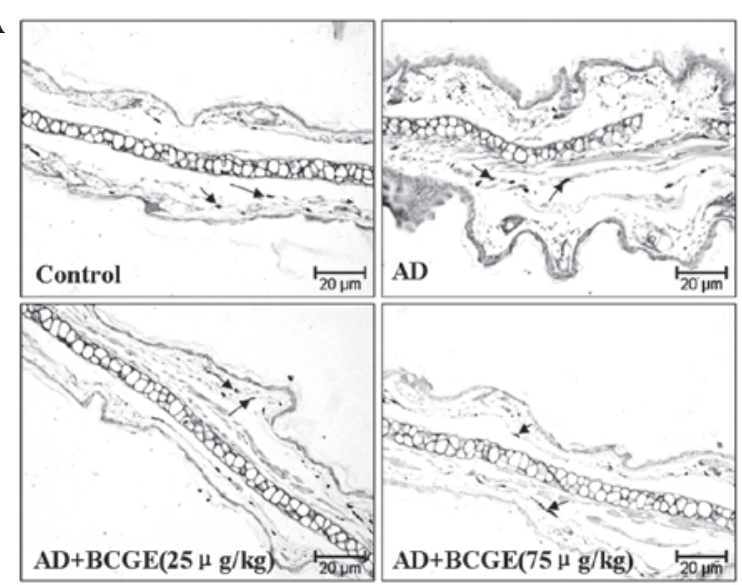

C

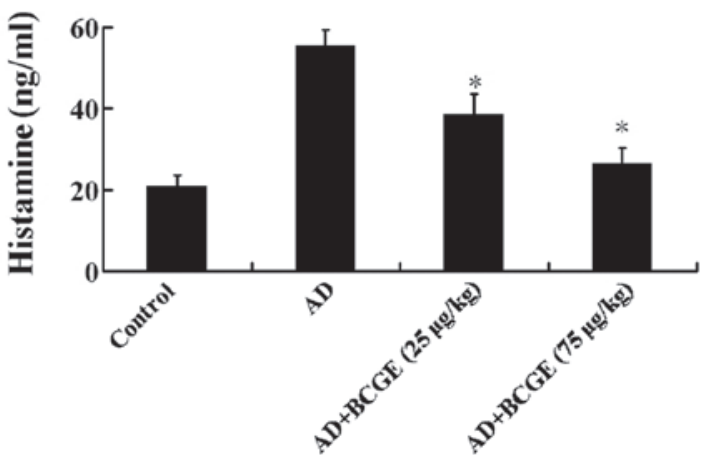

B

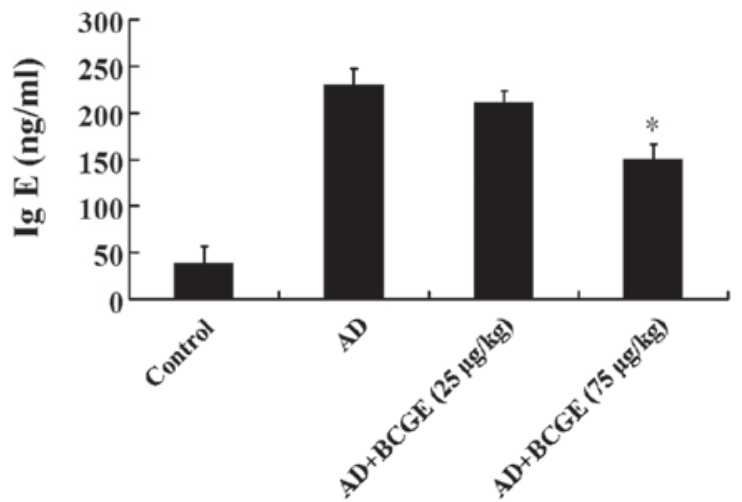

D

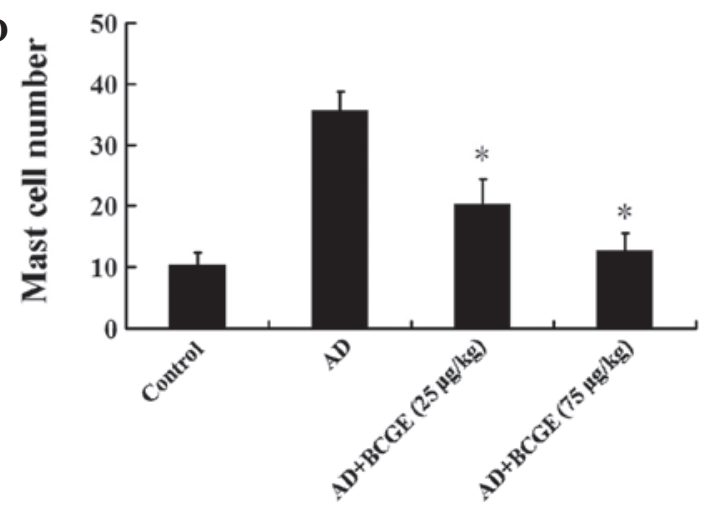

Figure 2. Effect of BCGE on mast cell infiltration, serum total IgE and histamine levels in BALB/c mice. (A) Ear sections were stained with toluidine blue. (B) The number of mast cells in five sites selected at random was counted with a microscope at a magnification of x200. (C) Levels of IgE and (D) histamine were measured in serum obtained from animals. The detailed experimental procedure is described in the materials and methods section. Values are expressed as the means $\pm \mathrm{SD} .{ }^{*} \mathrm{P}<0.05$ vs. control group. ${ }^{\prime} \mathrm{P}<0.05$ vs. AD group. BCGE, Bacillus Calmette-Guerin extract; IgE, immunoglobulin E; AD, atopic dermatitis. 

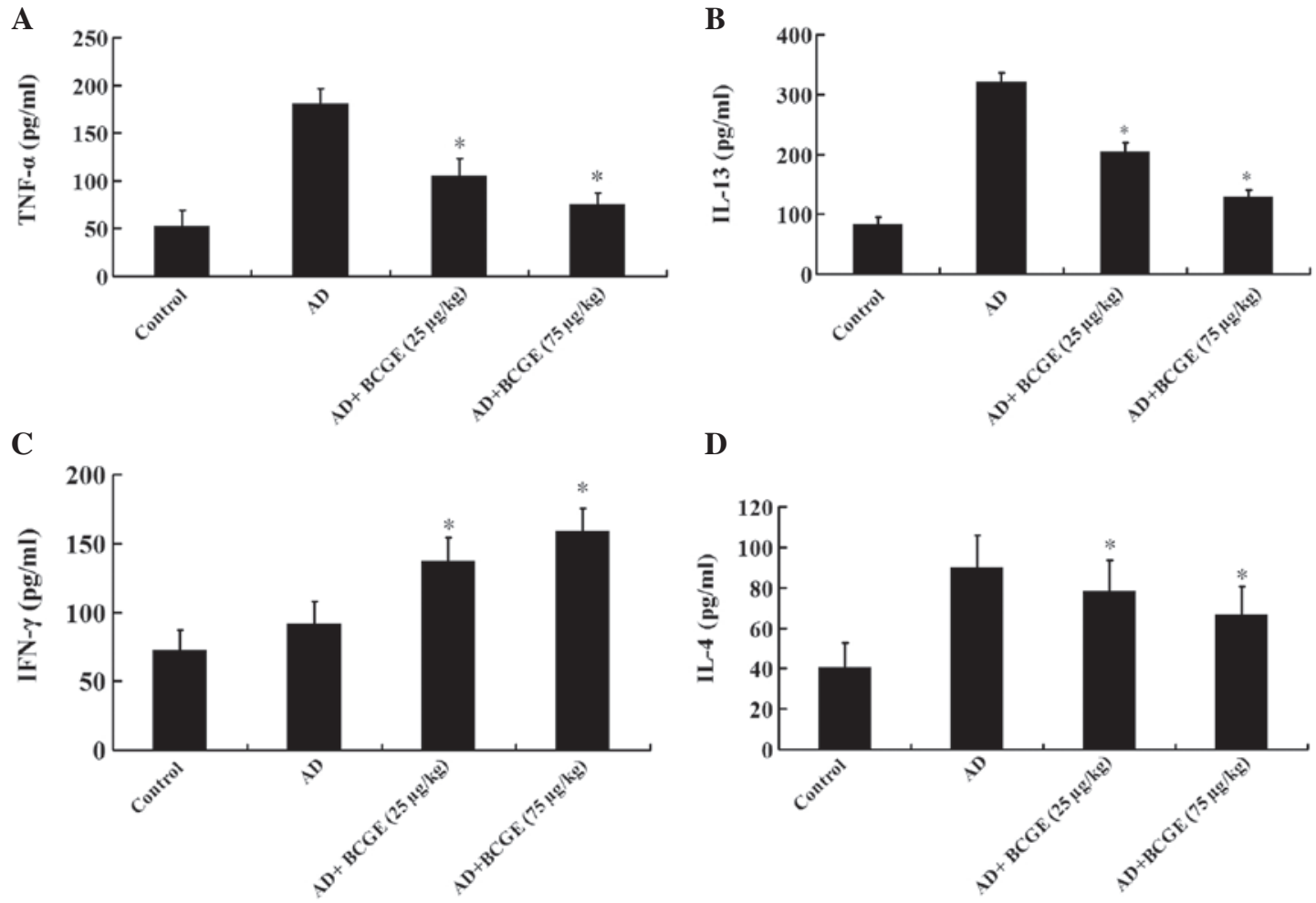

D

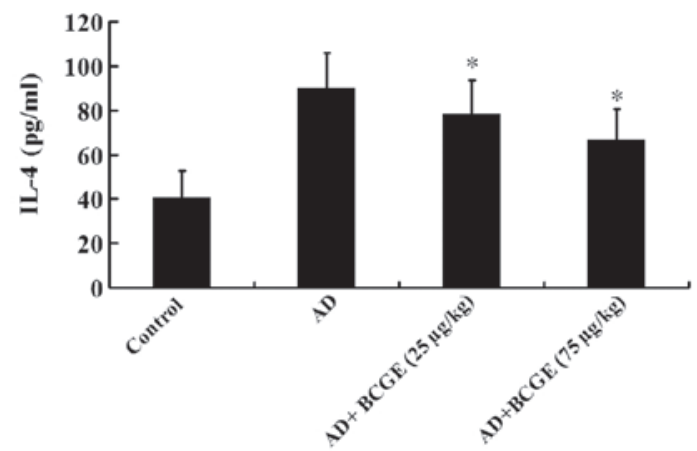

Figure 3. Effects of BCGE on cytokine levels in the ear. Levels of IL-4, IFN- $\gamma$, IL-13 and TNF- $\alpha$ were measured in ear biopsy homogenates. The detailed experimental procedure is described in the materials and methods section. Values are expressed as the means \pm S.D. ${ }^{*} \mathrm{P}<0.05$ vs. control group. ${ }^{*} \mathrm{P}<0.05$ vs. AD group. BCGE, Bacillus Calmette-Guerin extract; IL, interleukin; TNF- $\alpha$, tumor necrosis factor- $\alpha$; IFN- $\gamma$, interferon- $\gamma$; AD, atopic dermatitis.

cells induced by DNCB was suppressed by BCGE treatment. As shown in Fig. $2 \mathrm{C}$ and D, the level of IgE in the AD group was significantly higher than those in the control group $(\mathrm{P}<0.05)$. BCGE reduced the level of IgE in a dose-dependent manner. Similarly, the histamine level in the AD group increased compared with those in the control group, whereas the levels in the BCGE-treated group decreased compared with those in the control group. These results demonstrated that treatment with BCGE prevented the development of dermatitis in $\mathrm{BALB} / \mathrm{c}$ mice.

Effects of BCGE on cytokine levels in the ear. In order to gain better insights into the molecular mechanisms involved in the effect of BCGE on AD, IL-4, IL-13, IFN- $\gamma$ and TNF- $\alpha$ were measured in ear biopsy homogenates of BALB/C mice. The level of IL-4, IL-13 and TNF- $\alpha$ in the AD group was higher than those in the control group $(\mathrm{P}<0.05)$, while the level of IFN- $\gamma$ did not change significantly $(\mathrm{P}>0.05)$, the intramuscular application of BCGE markedly reduced DNCB-induced increases in IL-4, IL-13 and TNF- $\alpha$, while it elevated the IFN- $\gamma$ level in a dose-dependent manner (Fig. 3).

Effects of BCGE on NF- $\kappa B p 65$ expression in the nuclear extract of the ear tissue. Treatment of BCGE significantly downregulated the expression levels of IL-4, IL-13 and TNF- $\alpha$ (Fig. 3). Since NF-kB is one of the most common transcription factors involved in the expression of those cytokines $(14,15)$, we tested whether BCGE treatment is capable of reducing the NF- $\mathrm{\kappa Bp} 65$ expression in the nuclear extract of ear tissue by western blotting. Indeed, BCGE treatment significantly downregulated the NF- $\mathrm{kBp} 65$ expression in a dose-dependent

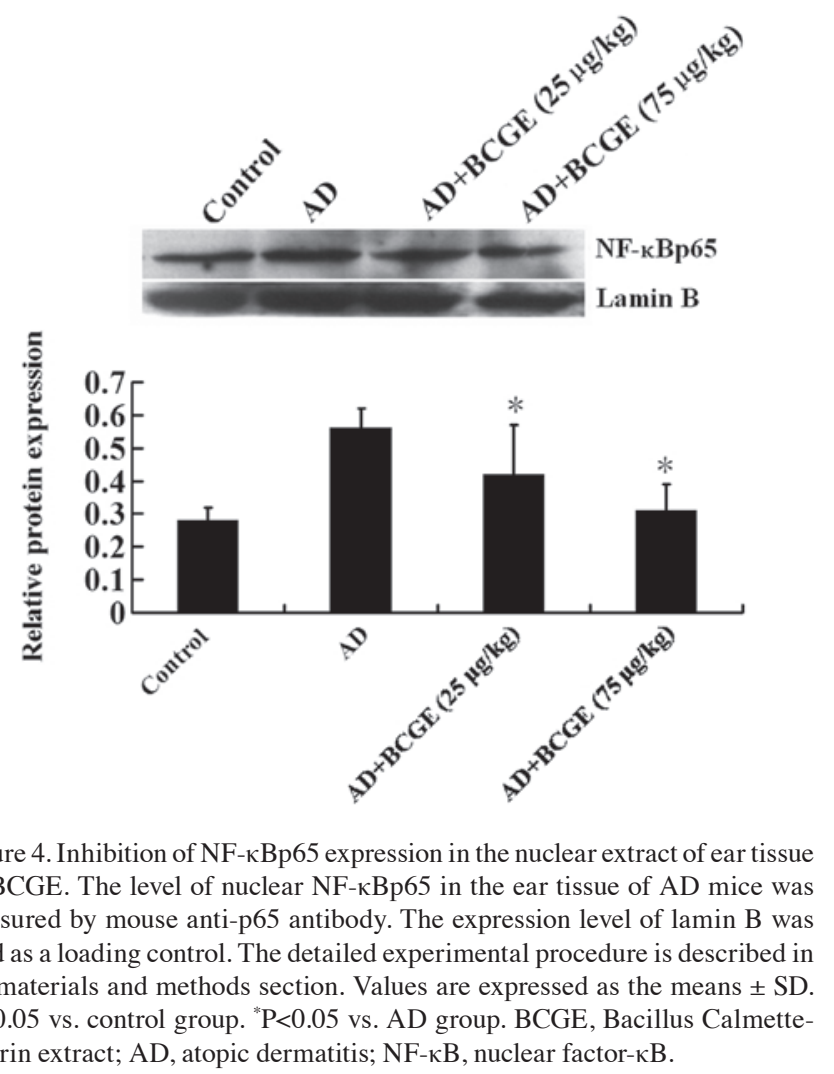

manner (Fig. 4). Data indicated that BCGE downregulated the levels of IL-4, IL-13 and TNF- $\alpha$, which may be due to downregulation of NF- $\kappa$ Bp65 expression. 


\section{Discussion}

In the present study, we investigated the effect of BCGE on a DNCB-induced AD model in BALB/c mice. We demonstrated that typical $A D$ was observed by the repeated alternative application of DNCB. Ear swelling and eczematous skin lesions were observed, and serum total $\operatorname{IgE}$ was increased. Histopathological analysis also revealed the infiltration of leukocytes, particularly mast cells, into the AD region. Intramuscular application of BCGE significantly inhibited these AD symptoms revealing the beneficial effect of BCGE on AD.

Mast cells are known as key effector cells in IgE-mediated allergic disorders and are activated by the cross-linking of a high affinity $\mathrm{IgE}$ receptor. Upon activation, mast cells undergo degranulation and release a variety of biologically active substances, which play an important role in host defense and allergic reactions, including AD. Among the immune mediators released from the mast cells, histamine is one of the best characterized and most potent $(16,17)$. In patients with AD, increases of histamine levels have been observed (18).

Infiltration of mast cells into the dermis is a necessary factor in order to define an appropriate animal model for AD (19). In the present study, we demonstrated that repeated treatment of DNCB resulted in mast cell infiltration into the dermis in the ear, suggesting that the animal model of AD used in this study satisfied AD conditions. Intraperitoneal application of BCGE significantly suppressed the infiltration of mast cells in the skin lesions of the AD mice and serum histamine release, suggesting that the activation and migration of mast cells may be a target of BCGE in AD.

Mast cell activation and histamine release are tightly regulated by IgE from B cells and serum IgE levels are elevated in proportion to the development of AD (19-21). In our study, DNCB-induced serum total IgE levels were reduced by BCGE. From these results, we assume that BCGE alleviates AD lesions through the inhibition of $\operatorname{IgE}$ production. IgE synthesis by $B$ cells is regulated by Th 2 cytokines, particularly IL- 4 and IL-13. In humans, overproduction of IL-4 is a critical factor in AD development (22). Sensitization to an allergen reflects the allergen's ability to elicit a Th2 cell response, in which IL-4 and IL-13 drive IgE production by promoting class-switch recombination in B cells (17). In our results, BCGE decreased IL-4 and IL-13, which are important in isotype switching to IgE. These results imply that BCGE is capable of reducing serum IgE by suppressing the Th2 response, in particular the production of IL-4 and IL-13.

The production of pro-inflammatory cytokines, particularly TNF- $\alpha$, by epidermal cells, is one of the key events in the initiation of AD (23). TNF- $\alpha$ produced at the initiation stage of $\mathrm{AD}$ induces the production of various chemokines/adhesion molecules, which causes the recruitment and proliferation of leukocytes within the skin. BCGE is known to possess antiinflammatory activities $(24,25)$. We previously demonstrated that the production of TNF- $\alpha$ was inhibited by BCGE in activated human mast cells (26). Therefore, we hypothesize that the inhibitory effect of BCGE on leukocyte infiltration in our AD model may be mediated by the blocking of TNF- $\alpha$ and downstream chemokines/adhesion molecules.
The NF- $\kappa \mathrm{B}$ transcription factor is important in a number of cellular processes, particularly in inflammation and tumor development. $\mathrm{NF}-\kappa \mathrm{B}$ is normally sequestered in the cytoplasm by a family of inhibitory proteins known as inhibitors of $\kappa B$ (I $\mathrm{B})$. A wide variety of stimuli cause the phosphorylation of I $\mathrm{B} \alpha$, which is followed by its ubiquitination and subsequent degradation. The loss of $\mathrm{I} \kappa \mathrm{B} \alpha$ results in the release of the free $\mathrm{NF}-\kappa \mathrm{B}$ unit $\mathrm{p} 65$, which translocates from the cytoplasm to the nucleus, where p65 induces the expression of numerous proinflammatory molecules (27). In our study, BCGE attenuated the expression of nuclear NF- $\mathrm{Bp} 65$ in the ear, which may explain its inhibitory effect on levels of cytokines, however, additional evidence is required.

In conclusion, the present study demonstrates that the intramuscular application of BCGE inhibits the development of DNCB-induced AD symptoms in BALB/c mice. The inhibitory effect of BCGE was mediated by inhibiting histamine release, the production of IgE and the level of IL-4, IL-13 and TNF- $\alpha$, while increasing the level of IFN- $\gamma$. The underlying mechanism of the therapeutic effect of BCGE is mainly mediated by a reduction of $\mathrm{NF}-\kappa \mathrm{Bp} 65$ transactivity. Our results suggest that BCGE may be a potential therapeutic candidate for AD.

\section{Acknowledgements}

This study was supported by the National Science Foundation, (grant no. 81173092/H3105)

\section{References}

1. Leung DY, Jain N and Leo HL: New concepts in the pathogenesis of atopic dermatitis. Curr Opin Immunol 15: 634-638, 2003.

2. Leung DY and Bieber T: Atopic dermatitis. Lancet 361: 151-160, 2003.

3. Choi EJ, Lee S, Hwang JS, Im SH, Jun CD, Lee HS and Kim SH: DA-9601 suppresses 2,4-dinitrochlorobenzene and dust mite extract-induced atopic dermatitis-like skin lesions. Int Immunopharmacol 11: 1260-1264, 2011.

4. Furue M, Terao H, Rikihisa W, Urabe K, Kinukawa N, Nose Y and Koga T: Clinical dose and adverse effects of topical steroids in daily management of atopic dermatitis. Br J Dermatol 148: 128-133, 2003.

5. Caramalho I, Lopes-Carvalho T, Ostler D, Zelenay S, Haury M and Demengeot J: Regulatory T cells selectively express toll-like receptors and are activated by lipopolysaccharide. J Exp Med 197: 403-411, 2003.

6. Yamamoto $S$, Kuramoto E, Shimada $S$ and Tokunaga T: In vivo augmentation of natural killer cell activity and production of interferon- $\alpha / \beta$ and $\gamma$ with deoxyribonucleic acid fraction from Mycobacterium bovis BCG. Jpn J Cancer Res 79: 866-873, 1988.

7. Yamamoto S, Yamamoto T, Kataoka T, Kuramoto E, Yano O and Tokunaga T: Unique palindromic sequences in synthetic oligonucleotides are required to induce INF and augment INF-mediated natural killer activity. J Immunol 148: 4072-4076, 1992.

8. Fujieda S, Iho S, Kimura Y, Sunaga H, Igawa H, Sugimoto C Yamamoto S and Saito H: DNA from Mycobacterium bovis bacillus Calmette-Guérin (MY-1) inhibits immunoglobulin E production by human lymphocytes. Am J Respir Crit Care Med 160: 2056-2061, 1999.

9. Zhu XL and Zhang Y: Pharmacological property and clinical application progress of BCG-polysaccharide nucleic acid. China Mod Med 20: 19-21, 2013 (In Chinese).

10. Fletcher $\mathrm{CH}$ and Crossgrove $\mathrm{R}$ (eds): Institute for Laboratory Animal Research: Guide for the Care and Use of Laboratory Animals. 8th Edition. The National Acadamies Press, Washington, DC, 2011. 
11. Kwon HK, Lee CG, So JS, Chae CS, Hwang JS, Sahoo A, $\mathrm{Nam} \mathrm{JH}$, Rhee JH, Hwang KC and Im SH: Generation of regulatory dendritic cells and $\mathrm{CD} 4^{+} \mathrm{Foxp}^{+} \mathrm{T}$ cells by probiotics administration suppresses immune disorders. Proc Natl Acad Sci USA 107: 2159-2164, 2010.

12. Matsuda H, Watanabe N, Geba GP, Sperl J, Tsudzuki M, Hiroi J, Matsumoto M, Ushio H, Saito S, Askenase PW and Ra C: Development of atopic dermatitis-like skin lesion with $\operatorname{IgE}$ hyperproduction in NC/Nga mice. Int Immunol 9: 461-466, 1997.

13. Yuan XY, Ma HM, Li RZ, Wang RY, Liu W and Guo JY: Topical application of aloperine improves 2,4-dinitrofluorobenzeneinduced atopic dermatitis-like skin lesions in NC/Nga mice. Eur J Pharmacol 658: 263-269, 2011

14. Perkins ND: Integrating cell-signalling pathways with $\mathrm{NF}-\kappa \mathrm{B}$ and IKK function. Nat Rev Mol Cell Biol 8: 49-62, 2007.

15. Dong J, Jimi E, Zeiss C, Hayden MS and Ghosh S: Constitutively active NF- $\kappa$ B triggers systemic TNF- $\alpha$ dependent inflammation and localized TNF- $\alpha$ independent inflammatory disease. Genes Dev 24: 1709-1717, 2010.

16. Galli SJ, Nakae S and Tsai M: Mast cells in the development of adaptive immune responses. Nat Immunol 6: 135-142, 2005.

17. Galli SJ, Tsai M and Piliponsky AM: The development of allergic inflammation. Nature 454: 445-454, 2008.

18. Yanagisawa R, Takano H, Inoue K, Koike E, Kamachi T, Sadakane $\mathrm{K}$ and Ichinose T: Titanium dioxide nanoparticles aggravate atopic dermatitis-like skin esions in $\mathrm{NC} / \mathrm{Nga}$ mice. Exp Biol Med (Maywood) 234: 314-322, 2009.

19. Matsuoka H, Maki N, Yoshida S, Arai M, Wang J, Oikawa Y, Ikeda T, Hirota N, Nakagawa $\mathrm{H}$ and Ishii A: A mouse model of the atopic eczema/dermatitis syndrome by repeated application of a crude extract of house-dust mite Dermatophagoides farinae. Allergy 58: 139-145, 2003.
20. Kim SH, Jun CD, Suk K, Choi BJ, Lim H, Park S, Lee SH, Shin HY, Kim DK and Shin TY: Gallic acid inhibits histamine release and proinflammatory cytokine production in mast cells. Toxicol Sci 91: 123-131, 2006.

21. Kang JS, Yoon WK, Han MH, Lee H, Lee CW, Lee KH, Han SB, Lee K, Yang KH, Park SK and Kim HM: Inhibition of atopic dermatitis by topical application of silymarin in NC/Nga mice. Int Immunopharmacol 8: 1475-1480, 2008.

22. Yamazaki F, Aragane Y, Maeda A, Matsushita K, Ueno K, Yudate T, Kawada A and Tezuka T: Overactivation of IL-4induced activator protein-1 in atopic dermatitis. J Dermatol Sci 28: 227-233, 2002.

23. Homey B, Steinhoff M, Ruzicka T and Leung DYM: Cytokines and chemokines orchestrate atopic skin inflammation. J Allergy Clin Immunol 118: 178-189, 2006.

24. Kim HS, Kundu JK, Lee JS, Oh TY, Na HK and Surh YJ: Chemopreventive effects of the standardized extract (DA-9601) of Artemisia asiatica on azoxymethane-initiated and dextran sulfate sodium-promoted mouse colon carcinogenesis. Nutr Cancer 60 (Suppl 1): 90-97, 2008.

25. Kim JY, Kim DY, Lee YS, Lee BK, Lee KH and Ro JY: DA-9601, Artemisia a siatica herbal extract, ameliorates airway inflammation of allergic asthma in mice. Mol Cells 22: 104-112, 2006.

26. Suh WM, Park SB, Lee S, Kim HH, Suk K, Son JH, Kwon TK, Choi HG, Lee SH and Kim SH: Suppression of mast-cellmediated allergic inflammation by Lindera obtusiloba. Exp Biol Med (Maywood) 236: 240-246, 2011.

27. Li Q and Verma IM: NF- $\kappa$ B regulation in the immune system. Nat Rev Immunol 2: 725-734, 2002. 